Case report

\title{
Primary multiple tumor with affection of the thyroid gland, uterus, urinary bladder, mammary gland and other organs
}

\author{
A. Romaniuk a, M. Lyndin a, , V. Smiyanov ${ }^{\text {b }}$, Vl. Sikora ${ }^{a}$, A. Rieznik ${ }^{a}$, Y. Kuzenko ${ }^{a}$, \\ H. Budko ${ }^{a}$, Yu. Moskalenko ${ }^{c}$, L. Karpenko $^{a}$, Vol. Sikora $^{d}$, O. Gladchenko $^{\text {e }}$ \\ a Sumy State University, Department of Pathology, Ukraine \\ ' Sumy State University, Ukraine \\ c Sumy State University, Department of Orthopedics, Traumatology and Emergency Conditions, Ukraine \\ d Sumy State University, Surgery Department with Pediatric Surgery and Urology, Ukraine \\ e Sumy State University, Foreign Languages Department, Ukraine
}

\section{A R T I C L E I N F O}

\section{Article history:}

Received 10 October 2016

\section{Keywords:}

Case report

Multiple primary tumor

Lynch syndrome

Cancer

Breast

Bladder

Thyroid

Uterus

\begin{abstract}
A B S T R A C T
Background: Nowadays multiple primary tumor is characterized by growth and development of two or more tumors in one patient. The total world sickness rate ranges from $1 \%$ to $37 \%$. The presence of four or more tumors in one patient is rare case and presented as casuistry.

Case presentation: We showed a case of multiple primary tumor with metahronic lesion of the thyroid, uterus and breast, followed by synchronous benign tumors of the subcutaneous fat, urinary bladder and gallbladder were considered. The development of all malignant tumors in all cases was accompanied by the presence of benign precancerous processes. Analysis of neoplasia histology shows the predominance of poorly differentiated forms of cancers in women with increased aggressiveness of cancerous tissue in each subsequent case and the growth of metastatic ability. The influence of heredity on the tumors progress is confirmed by immunohistochemical characteristics of cancer cells. Steroid-sensitive tissue of the uterus and breast in both cases didn't express ER and PR, in all cases the tissue had overexpression of Ki-67, p53, bax and bcl-2 receptors. The results of DNA testing for determination the Lynch syndrome revealed the presence of microsatellite instability in genetic material. The results of studies revealed the absence of mutations in these genes (MLH1, MSH2 and MSH6). Despite the negative results of the study, it doesn't exclude the possibility of Lynch syndrome for $100 \%$, and its presence may be caused by the mutations of other genes (PMS1, PMS2 and MLH3), responsible for DNA repair. Unfortunately there wasn't any opportunity to study their mutations.

Conclusions: While studying the anamnesis of life and disease of women it was revealed that she had multiple primary tumor with lesions of the breast, urinary bladder, thyroid, uterus and other organs. This study shows that neoplastic tissue in all cases had high rates of cell proliferation, their antiapoptotic stability, expression of prognostically unfavorable-receptors, and absence of favorable prognostic markers. Histological study revealed high rates of malignant neoplastic tissue. It indicates to the existence of common mechanisms of malignant tumors and their genetic predisposition that can be clearly observed in many generations of patient.
\end{abstract}

(c) 2017 Elsevier GmbH. All rights reserved.
Abbreviations: MPT, Multiple primary tumor; ICD, International Statistical Classification of Diseases.

* Corresponding author.

E-mail addresses:

pathomorph@gmail.com (A. Romaniuk), n.lyndin@med.sumdu.edu.ua (M. Lyndin), v.smiyanov@med.sumdu.edu.ua (V.Smiyanov), vlad-sikora@ukr.net

(Vl. Sikora), a.reznik@med.sumdu.edu.ua (A. Rieznik),kuzenko_yevhen@rambler.ru (Y. Kuzenko), ay.budko@med.sumdu.edu.ua (H. Budko),

yl.moskalenko@med.sumdu.edu.ua (Yu. Moskalenko),

l.karpenko@med.sumdu.edu.ua (L. Karpenko),v.sikora@med.sumdu.edu.ua

(Vol. Sikora), oksanagl123@gmail.com (O. Gladchenko).

http://dx.doi.org/10.1016/j.prp.2017.01.003

0344-0338/@ 2017 Elsevier GmbH. All rights reserved.

\section{Introduction}

The first documented case of a primary multiple tumor (PMT) was found in the works of Avicenna. He described bilateral affection of mammary glands. In 1793 John Pierce described a patient who consistently had cancer in the right and then in the left mammary gland and then uterus cancer. Billroth made special contribution to the study of this issue. In 1869 he identified the following requirements to PMT: tumors should be located in different organs, they should have different morphological structure, and each tumor 
must give its own metastases. In 1932, Warren and Gates published the first study in 1259 patients with PMT [1].

PMT is now understood as an independent appearance and development of two or more tumors that arise simultaneously (synchronous PMT) or at regular intervals (metachronous PMT) in one patient. The total morbidity in the world ranges from $1 \%$ to $37 \%$, depending on the type of used analyses, the time of carrying out the investigations and the ethnicity of patients [2-4]. Cases of triple localization occur in $5-8 \%$ of cases. The presence of four or more tumors in one patient is a rare case and is considered as a casuistry [5].

There is no common statement of PMT appearance reasons in scientific reports [6]. Age, gender, ethnicity, obesity, smoking, topography and histological type of tumor, its pre-treatment, the impact of the environment are described among these reasons [7]. But genetic predisposition [8] is recognized to have the most significant influence on the PMT appearance. It is manifested by the presence of certain genes (BRCA1, BRCA2, p16/CDKN2A, hMSH2, hMLH1) and their polymorphism. These genes are responsible for proapoptotic and antiapoptotic function of cells [7-9]. Data about the participation of P53 gene and its mutations in multiple tumor genesis were repeatedly highlighted in publications. We can not exclude the multi-faceted impact of P53 gene on the initiation of carcinogenesis $[10,11]$.

There are cases in which the cause of PMP is a disorder in the system of DNA reparation. Lynch syndrome is one of such examples. It is a hereditary disease that occurs in case of a mutation in one gene of DNA mismatch repair (MMR) [12]. It is characterized by the presence of microsatellite instability and mutations in MLH1, MSH2, MSH6, PMS1, PMS2 and MLH3 genes [13].

The survival of patients with PMT varies and depends on many factors (localization of the primary center, synchronism or metahronnism of a tumor etc.). More and more data about the survival of patients with PMT appears in the reports, comparatively with patients who have a neoplasia of one localization [14]. It is connected with the presence of more differentiated forms of cancers in case of PMT which requires less aggressive therapy.

\section{Case presentation}

A 62-year old woman was born in Russian city of Voronezh in 1953. Her family lived near a chemical plant, where her parents worked throughout their lives. From anamnesis we have known that her grandfather (on her father's side), her father and brother died of malignant neoplasms in the intestines at the age of under 50. Also we have known that her uncle (on her mother's side) died of urinary bladder cancer at the age of 61 .

The woman was treated for such non-neoplastic diseases as pneumonia, chronic obstructive pulmonary disease, hastropathy, chronic tonsillitis, hepatosis and cystitis throughout her life.

For the first time our patient addressed doctors concerning the neoplasm of subcutaneous fat at the age of 16 in 1969, and it was only the beginning of her oncological sickness.

\subsection{Forearm tumor}

The neoplasm of left forearm was removed in patient at the age of 16 in 1969. This tumor was relapsed twice in 1985 and 2015. In the latter case, the tumor had oval shape, it was of yellow colour with hemorrhages, and its size was $6 \times 4 \times 3 \mathrm{~cm}$. The tumor was represented by typical adipose tissue surrounded by a thin fibrous capsule. In all cases, left forearm lipoma (International Statistical Classification of Diseases 10 (ICD) - D17.2) was diagnosed after histopathological study.

\subsection{Thyroid tumor}

For the first time hypoechoic nodular formation on the front surface of the right part of the thyroid gland was revealed during the ultrasound investigation in 2008. Its size was $16 \times 13 \times 24 \mathrm{~mm}$. On the back surface of the same part of the thyroid gland isoechoic formation with $11 \times 8 \times 14 \mathrm{~mm}$ hydrophilic rim was revealed. As a result nontoxic multinodular goitre of the 1 st stage was diagnosed in our patient (ICD 10 - E04.2). We have known that our patient's mother also suffered from goitre of the thyroid gland.

This disease has eventually progressed:

- additional isoehoic formation with a hydrophilic rim was revealed in the right part of the thyroid gland in 2009. Its diameter was $5 \mathrm{~mm}$;

- the center which size was $16 \times 13 \times 24 \mathrm{~mm}$, increased to $16 \times 17 \times 28 \mathrm{~mm}$ in 2011 and eventually it obtained moderately reduced echogenic lobulated structure in 2013;

- the center on the back surface of the right part of the thyroid gland which size was $11 \times 8 \times 14 \mathrm{~mm}$, increased to $12 \times 10 \times 16 \mathrm{~mm}$. Hydrophilic area without clear contours appeared at the periphery. Its size was $8 \times 6 \times 5 \mathrm{~mm}$;

- hypoechoic formation with irregular and indistinct contours appeared on the back surface of the left part of the thyroid gland. Its size was $10 \times 8 \times 16 \mathrm{~mm}$;

- a reaction from anterior jugular lymph nodes appeared on both sides. These lymph nodes with preserved echoic structure increased to $13 \times 5 \mathrm{~mm}$.

The results of puncture biopsy research of the thyroid gland lumps also had negative dynamics. In 2012 cytogram corresponded to a nodular goitre. In 2013 cytogram of punctates corresponded to a follicular neoplasia of the thyroid gland. There were layers and groups of epithelial cells with signs of atypia in the received material; they were presented in the form of microfollicular structures.

Surgeons performed subtotal resection of the thyroid gland with the purpose of treatment. Histological examination revealed that the tumor had been presented with tumor cells in the form of solid clusters with a single follicles formation. Follicular cells had various degrees of cellular atypia, there was invasion into blood vessels and surrounding tissues (Fig. 1A). Follicular thyroid cancer, stage III T3NOMO (ICD 10 - C73) was diagnosed after intraoperative and histopathological investigation.

\subsection{Uterus tumor}

As a result of the uterine bleeding fractional scrapping of the walls of the uterus cavity was made in patient for diagnostic and therapeutic purposes in 2011 (the patient has had menopause since 2006). Endometrial glandular hyperplasia (ISD 10 - N85.0) was diagnosed. There was a relapse of bleeding 5 months later the same year. The results of histopathological study showed the growth of moderately varied adenocarcinoma in the uterus cavity. Hysterectomy with adnexas was performed in woman according to the health reasons. The results of macroscopic investigation showed that the uterus was not enlarged. When the uterus was cut an endometrial tumor $(3 \mathrm{~cm}$ in diameter) was revealed in the right corner. Left uterine tube was in the form of a tumor, which was not united with the outer surface of the uterus. Histological examination of endometrial tumor showed its growth. The tumor consisted of glandular-papillary structures with the presence of solid areas. Tumor cells were polymorphic, hyperchromic, there were numerous mitotic figures (Fig. 1B). The investigation of the left fallopian tube tissue revealed the presence of tumor growth from the side of adventitia. That tumor had the same histologic characteristics as the uterus tumor. Moderately varied uterine adenocarcinoma 


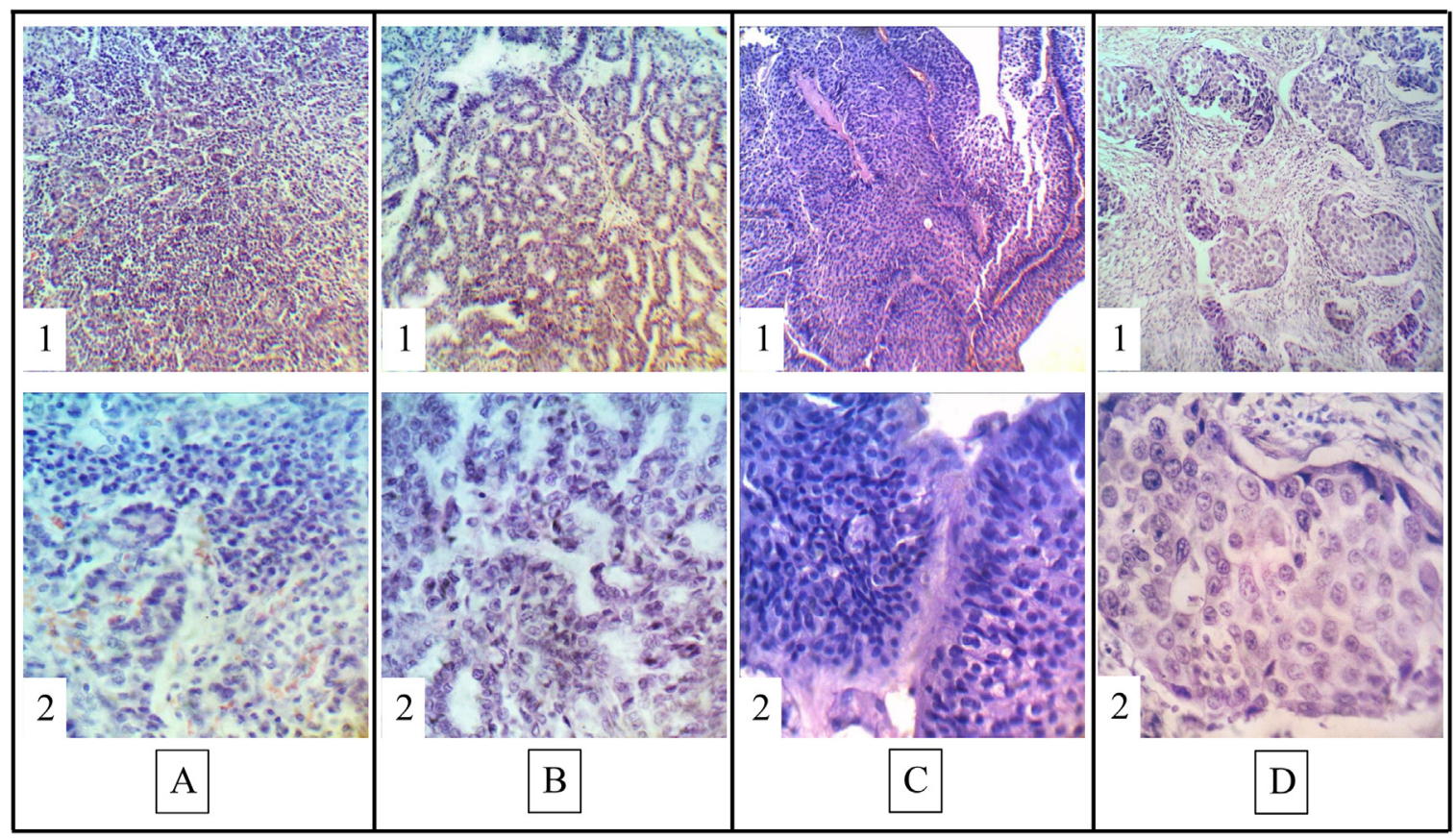

Fig. 1. A - Thyroid cancer, B - Uterus cancer, C - Urinary bladder papilloma, D - Breast cancer. Staining with hematoxylin and eosin. Enlargments -1 - $\times 100,2-\times 400$..

with metastases in the left fallopian tube, stage IIIa, T3aNOM0 (ICD 10 - C54.1) was verified after intraoperative and histopathological investigation.

\subsection{Urinary bladder tumor}

In 2015 , the woman felt the pain in the projection of the urinary bladder. Also she experienced frequent urge to urinate and had blood in the urine. The lump on the wall of the urinary bladder was found as a result of the examination. After transurethral resection of the tumor it was histopathologically investigated and it appeared to be introduced with transitional epithelium which had increased proliferative activity somewhere. Circular masses of monomorphic transitional epithelium with distinct contours, limited to one layer of basal cells, lay in the spongy soft fibrous stroma (Fig. 1C). Transitional cellular papilloma of the urinary bladder (ICD 10 - D30.3) was diagnosed.

\subsection{Mammary gland tumor}

In 1999 a painful lump in the right mammary gland was found in woman and she experienced sectoral resection of the right mammary gland. After intraoperative and histopathological investigation the following diagnosis was verified: cystic mastopathy with epithelial proliferation (ICD 10 - N60.3).

In 2015 ultrasound investigation revealed an irregular-shaped solid hypoechoic lump in the upper-inner quadrant of the right mammary gland. Its size was $1.9 \times 1.3 \mathrm{~cm}$. It had a lot of small calcifications in the internal structure. Axillary lymph nodes were dense, in the form of conglomerates and their size was up to $2.7 \mathrm{~cm}$ in diameter. Cytological examination showed the presence of glandular cancer cells. Halstead mastectomy was conducted on the right. When the tumor was cut it appeared to be of white-grey colour, dense, without a capsule. Hematoxylin and eosin staining of the tumor showed almost complete absence of tubular-glandular structures, evident cellular and nuclear atypia, a large number of abnormal mitoses, multi-nuclei and nuclear hyperchromatosis. Tumor tissue consisted of large cells that had lost the opportunity to typical distribution of the epithelium in the form of layers, but had tended to retain adhesion. Secondary changes were occured in the form of inflammation and necrosis in the tumor tissue (Fig. 1D). Tumor growth was also detected in lymph nodes taken during the operation. Infiltrative ductal carcinoma of the right mammary gland with a low degree of differentiation with metastases in 10 regional lymph nodes, stage IIB, T2N1M0 (ICD 10 - C50.2) was diagnosed after intraoperative and histopathological investigation. A month later, a lump was detected in the lower third of the neck on the right during the ultrasound investigation of the patient's neck. Its size was $12 \times 10 \times 9 \mathrm{~mm}$ in diameter. It was regarded as a lowgrade adenocarcinoma metastasis of mammary gland cancer to the lymph nodes of the neck. It was verified cytologically.

\subsection{Gallbladder tumor}

Doctors revealed a polyp (ICD 10 - K82.8) on the back wall of the gallbladder of the patient during preventive ultrasound investigation of the abdominal cavity organs in 2009. Its diameter was $3 \mathrm{~mm}$. It has become $2 \mathrm{~mm}$ bigger for six years.

\subsection{Immunohistochemical investigation of tumors}

We performed immunohistochemical investigation of receptors in neoplasms tissue in order to establish the sensitivity to therapy, to detect the degree of malignancy and to evaluate proliferative-apoptotic potential of tumors. Receptors to estrogen (ER), progesterone (PR), second type receptors of human epidermal growth factor (HER2/neu) and Ki-67 were established using monoclonal rabbit antibodies (SP1, YR85, SP3 and SP6 clones respectively). Receptors to $\mathrm{p} 53$, bcl-2 and bax were established using monoclonal mice antibodies (SP5, 100/D5 and 2D2 clones respectively).

Thyroid cancer tissue has high rates of expression of Ki-67 and p53 proteins (70\% of cells) and (45\% of cells) respectively. Positive cytoplasmic reaction on the availability of antiapoptotic bcl-2 receptors is found in $85 \%$ of neoplastic cells. Follicular cancer cells on the membrane and in cytoplasm express receptors to proapoptotic bax proteins (Fig. 2-2). 


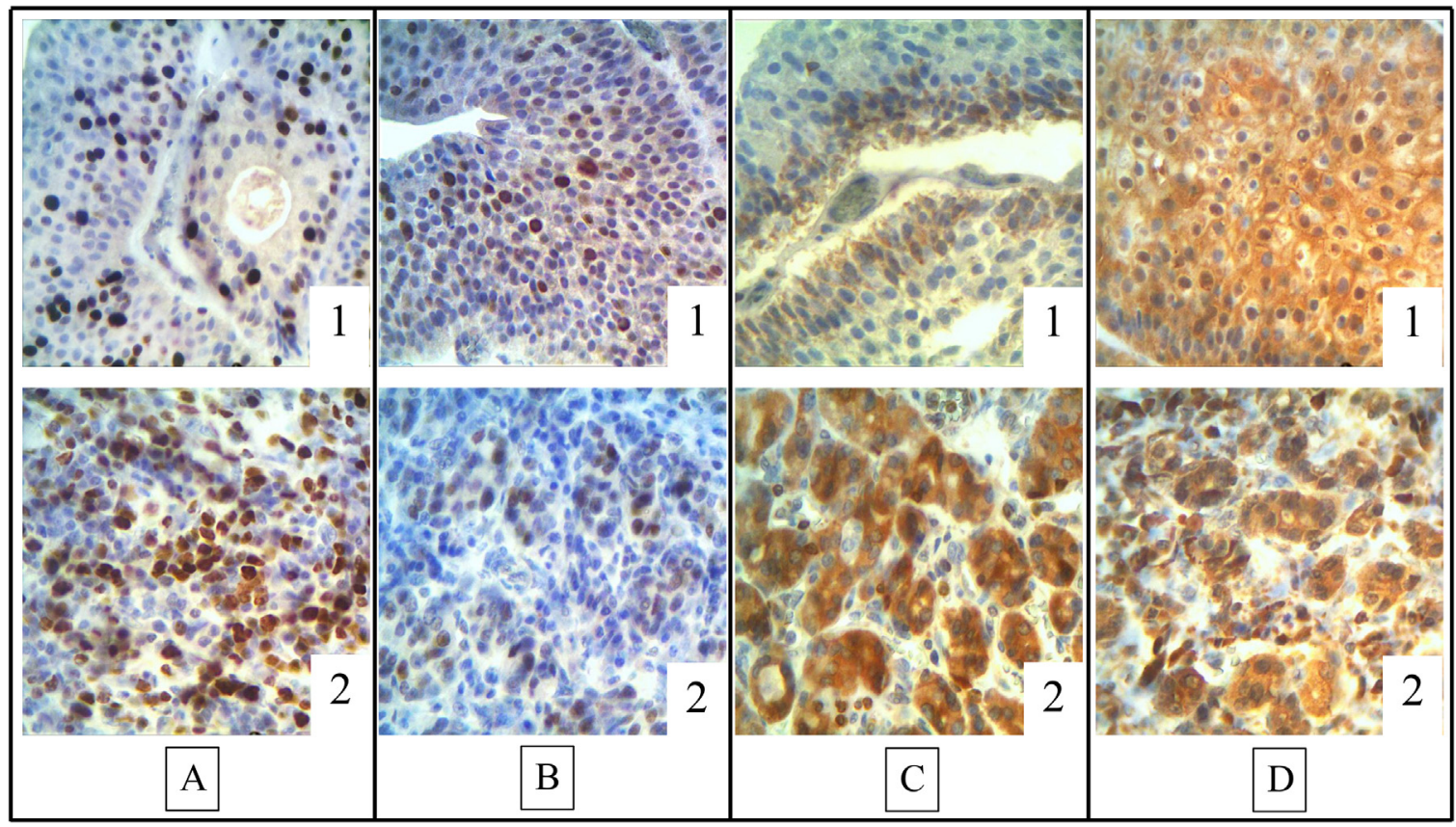

Fig. 2. 1. - Urinary bladder papilloma. 2. - Thyroid cancer. A - Ki-67, B - p53, C - bcl-2, D - bax. Immunohistochemical study of receptors. Enlargment $\times 400$.

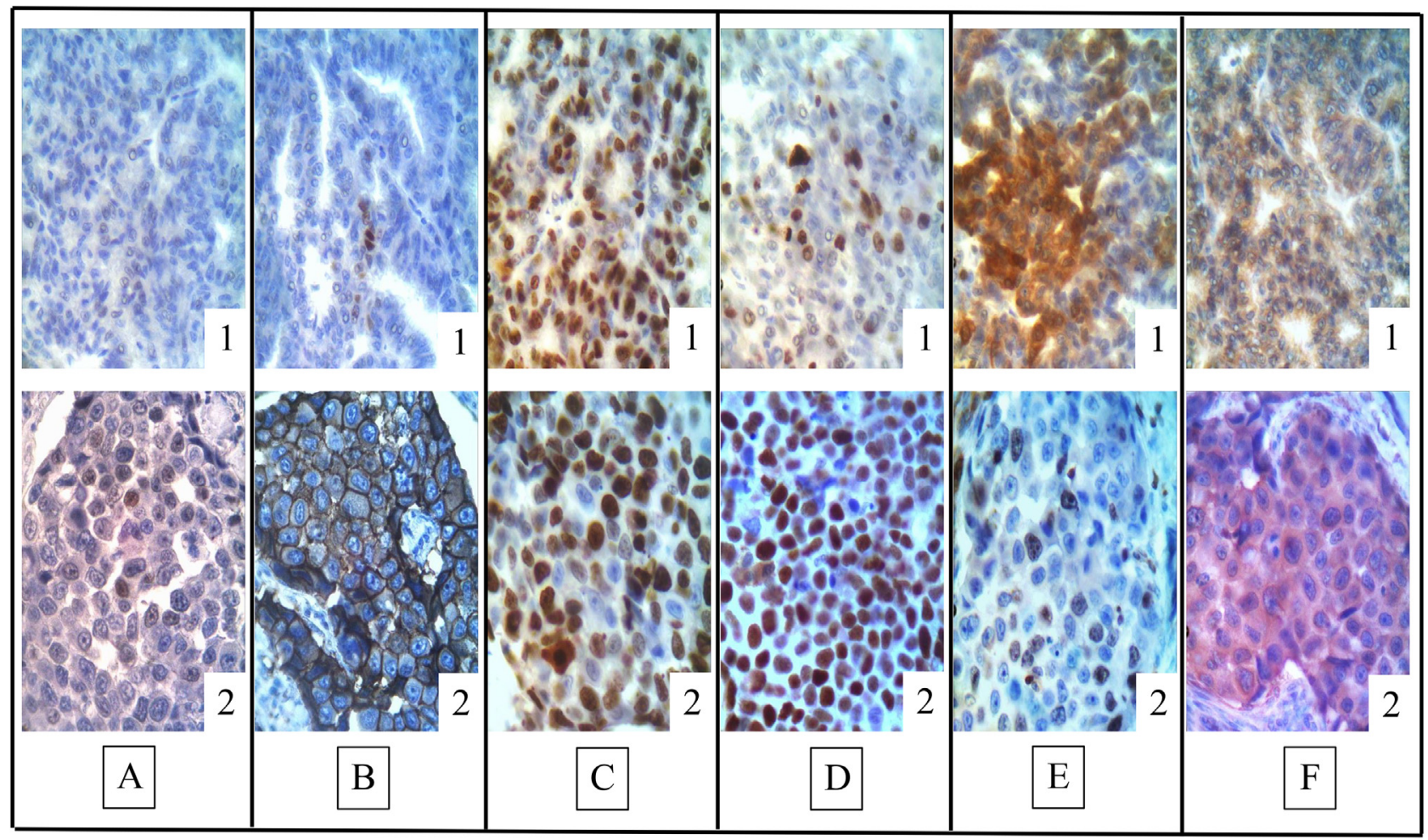

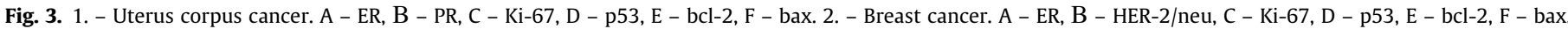
Immunohistochemical study of receptors. Enlargment $\times 400$.

Isolated centers of cells with ER expression (7\% of cells) and with PR expression ( $4 \%$ of cells) are detected in uterine adenocarcinoma tissue on the background of almost complete absence of steroidpositive tumor profile. High rates of cells proliferative activity (K-67 expression 80\%) and their antiapoptotic property (p53 expression $35 \%$ ) are revealed in the uterus carcinoma similar to the primary tumor in the thyroid gland. $50 \%$ of cells has positive immunohis- tochemical reaction to bcl-2 protein. Moderate positive reaction is observed in relation to the expression of bax receptors (Fig. 3-1).

The nuclei of transitional cellular epithelium of urinary bladder have a positive staining in relation to K-67 receptors (50\% of nuclei) and they also have positive staining in relation to p53 receptors ( $35 \%$ of nuclei). Weak positive reaction (20\%) is found on bcl-2 and bax receptors in the papilloma cells of urinary bladder (Fig. 2-1). 
Mammary gland cancer cells have overexpression of p53 receptors $(95 \%)$ and $\mathrm{Ki}-67$ receptors (80\%). But the reaction as to the availability of ER and PR is negative in neoplastic cells. Bcl-2 protein is not detected in the cytoplasm although single cells have positive nuclear reaction. Immunohistochemical reaction establishes the presence of receptors to bax protein and HER2/neu (Fig. 3-2).

We suggested the presence of Lynch syndrome in woman taken into consideration the patient's history of life. Her men relatives had malignant intestines disease in three generations, there was genetic predisposition to cancer in four generations. The woman also had PMT with affection of uterus at a young age. In order to confirm the diagnosis the investigation of microsatellite instability was carried out using fragmentary analysis with the help of five mononucleotide markers: NR21, NR24, NR27, BAT25 and BAT26. Having established the presence of microsatellite instability we amplificated all 45 coding exons of MLH1, MSH2 and MSH6 genes with associated parts of introns (50-100 nucleotide pairs) in selected DNA using 53 pairs of primers. We conducted the research of these genes mutations with the help of polymerase chain reaction techniques, conformational-sensitive electrophoresis and direct sequencing. DNA violations at Lynch syndrome are concentrated in these genes in $90 \%$ of cases. The results of research have found no mutations in MLH1, MSH2 and MSH6 genes.

\section{Discussion}

There is PMT with metachronous affection of the thyroid gland, uterus and mammary gland in our case. This PMT was accompanied with synchronous non-malignant affection of the subcutaneous tissue, urinary bladder and gallbladder. This disease is quite common disorder with diverse number of etiological factors which declares itself with the affection of various organs with tumor process $[4,5,7]$. In our case, there was a combination of several triggering factors: the impact of labor and living conditions of the woman and her inheritance, which are known [8] to have the most significant effect on the initiation and progression of carcinogenesis. The first factor affected not so much the woman-patient, but her parents, who had lived and worked in the conditions of harmful impact of chemical production. The second factor, in the form of inheritance, became apparent through the presence of malignant tumors in the anamnesis at relatives from the male line. Moreover, both patient's daughters have already had oncological problems at the time of the woman's interview. Her first daughter has a nodal lump in the thyroid gland and multiple papillomatosis of the skin and the second daughter has a cyst in her right mammary gland. This confirms the fatal influence of the inherited genetics on the possibility of malignant neoplasias for both daughters in future.

The results of DNA testing to identify Lynch syndrome have shown that microsatellite instability is present in the genetic material. But this feature is typical not only for Lynch syndrome [15]. This stimulated us study mutations in MLH1, MSH2 and MSH6 genes, which are often responsible for this destabilization of genome. Although the results of the investigation were negative, this does not exclude the possibility of Lynch syndrome in woman. The presence of Lynch syndrome may be connected with mutations in other genes (PMS1, PMS2 and MLH3), which are responsible for DNA reparation. Unfortunately, we had no opportunities to study these genes' mutations.

The analysis of histological situation of neoplasias shows the predominance of low differentiated forms of cancer (the most common malignant tumors of the uterus, thyroid, breast cancer in Ukraine) in woman [16,17]. There are tumors of moderate and high degree of malignancy with increased aggressiveness and the growth of metastatic ability of cancerous tissue in each subsequent accident. This is an indicator of the future progressive imbalance of the genetic material of the patient and its manifestation on cancer histogenesis.

General characteristics of cancerous cells immunohistochemical profile confirm the influence of heredity on the progress of tumor processes. Steroid-sensitive tissue of the uterus and mammary gland did not express ER and PR in both cases. The tissue had overexpression of Ki-67, p53, bax and bcl-2 receptors in all cases. The presence of HER2/neu in the cancer tissue of mammary gland indicates the tumor aggressiveness and the autogenic ability to support the neoplasia growth. High levels of antiapoptotic p53 and bcl-2 proteins expression in the urinary bladder tissue are the indicators of the possibility of its degeneration into cancer and the need for timely surgical treatment.

We observe the following consistency of heredity influence on the development of neoplasias taking into consideration all identified patient's tumors immunophenotype. Various gene mutations arise in cells under the influence of diversified factors namely p53 gene mutation, which has expressed carcinogenic effect [18]. Perhaps this happened to woman's ancestors and was transmitted to her hereditarily. Moreover we do not exclude the accumulation of this gene mutation in patient herself. The presence of mutant p53 gene eliminates the effects of wild-type p53 protein. This protein is responsible for cells apoptosis in the form of stimulating proapoptotic genes transcription, including bax. It is also responsible for blocking the antiapoptosis genes (bcl-2) expression. All this leads to a lack of defense of an organism against cells able to malignant transformation [19]. As a result it leads to uncontrolled cells division and strengthening of their proliferative activity (increase in the expression of $\mathrm{Ki}-67$ ) and apoptosis block (increase in the expression of bcl-2) [20]. Uncontrolled cells division, the absence of their elimination in the form of apoptosis, possible transformation into cancer cells and progression of malignancy accompany all of the mentioned above. The presence of proapoptotic bax protein is an indicator of body struggle against neoplastic processes. But its effect is eliminated due to the presence of bcl-2 proteins that form bcl-2-bax heterodimers, which prevent apoptosis [21].

It should be noted that the development of malignant tumors in all cases was accompanied by the presence of benign precancerous processes (nontoxic multinodular goitre, endometrial glandular hyperplasia and cystic mastopathy with epithelial proliferation). This indicates that the development of malignant neoplasias could be prevented by removing benign tumors in advance. Next generation of woman's children should focus on a high probability of a malignant tumor appearance, taking into consideration the fact that they have already had early signs of neoplasias development.

However, it should be noted that the woman has quite a good appearance and satisfactory health condition despite of a large number of survived operations and treated malignant tumors and even metastatic affection of the body. It was also observed in other cases with PMT [14]. This may indicate the adaptation of the macroorganism of the patient to the struggle against disastrous diseases.

\section{Conclusions}

Having studied the anamnesis of woman's life and disease we found that she had primary multiple tumor with the affection of mammary gland, urinary bladder, thyroid gland, uterus and other organs. These studies show that neoplastic tissue in all cases had high rates of cells proliferative activity and their antiapoptotic stability. This neoplastic tissue also had the availability of the expression of prognostically unfavorable receptors and the absence of prognostically favorable markers. High rates of neoplastic tissue malignancy were revealed during histological examination. This indicates the existence of common mechanisms of malignant 
tumors development and their genetic predisposition that can be clearly seen in many generations of our patient.

Data analysis of additional methods of examination shows that one of the key points in tumor development is the mutation of genes, p53 gene in particular. P53 gene mutation led to the escaping of cancer cells from the regulatory unit, which is responsible for cells division and progression of malignancy. We can not exclude the role of mismatch repair system disorder, although we have not confirmed mutations in the MLH1, MSH2 and MSH6 genes.

Complex investigation of patients, timely treatment of precancerous states and awareness of the mechanisms of rare diseases development can prevent permanent and progressive neoplastic processes in humans. This case can become an example which confirms all mentioned above.

\section{Competing interests}

The authors declare that they have no competing interests.

\section{Consent for publication}

Written informed consent was obtained from the patient for publication of this Case report and any accompanying images. A copy of the written consent is available for review by the Editor of this journal.

\section{Availability of data and materials}

The main results of histological and immunohistochemical studies presented in figures and text of this article. Genetic research results obtained in Coloproctology Research Center (Moscow).

\section{References}

[1] S. Warren, O. Gates, Multiple primary malignant tumors: a survey of the literature and statistical study, Am. J. Cancer 16 (1932) 1358-1414.

[2] A.B. Mariotto, J.H. Rowland, L.A. Ries, S. Scoppa, E.J. Feuer, Multiple cancer prevalence: a growing challenge in long-term survivorship, Cancer Epidemiol. Biomarkers Prev. 16 (3) (2007) 566-571.

[3] H.K. Weir, C.J. Johnson, T.D. Thompson, The effect of multiple primary rules on population-based cancer survival, Cancer Causes Control 24 (6) (2013) $1231-1242$

[4] Magid H. Amer, Multiple neoplasms, single primaries, and patient survival, Cancer Manage. Res. 6 (2014) 119-134.
[5] A. Salem, R. Abu-Hijlih, F. Abdelrahman, et al., Multiple primary malignancies: analysis of 23 patients with at least three tumors, J. Gastrointest. Cancer. 43 (3) (2012) 437-443

[6] S. Rosso, L. Terracini, F. Ricceri, R. Zanetti, Multiple primary tumours: incidence estimation in the presence of competing risks, Popul. Health Metr. 7 (2009) 5.

[7] I. Soerjomataram, J.W. Coebergh, Epidemiology of multiple primary cancers, Methods Mol. Biol. 471 (2009) 85-105.

[8] F. Albright, C. Teerlink, T.L. Werner, L.A. Cannon-Albright, Significant evidence for a heritable contribution to cancer predisposition: a review of cancer familiality by site, BMC Cancer 12 (2012) 138.

[9] M. Artac, H. Bozcuk, M. Ozdogan, et al., Different clinical features of primary and secondary tumors in patients with multiple malignancies, Tumori 91 (4) (2005) 317-320.

[10] M. Miyaki, T. Iijima, M. Ohue, Y. Kita, T. Hishima, T. Kuroki, T. Iwama, T. Mori, A novel case with germline p53 gene mutation having concurrent multiple primary colon tumours, Gut 52 (February (2)) (2003) 304-306.

[11] M.T. Rens, E.J. Eijken, J.R. Elbers, J.W. Lammers, M.G. Tilanus, P.J. Slootweg, p53 mutation analysis for definite diagnosis of multiple primary lung carcinoma, Cancer 94 (January (1)) (2002) 188-196.

[12] Lynch HT1, P.M. Lynch, S.J. Lanspa, C.L. Snyder, J.F. Lynch, C.R. Boland, Review of the Lynch syndrome: history, molecular genetics, screening, differential diagnosis, and medicolegal ramifications, Clin. Genet. 76 (July (1)) (2009) $1-18$.

[13] H. Vasen, G. Möslein, A. Alonso, et al., Guidelines for the clinical management of Lynch syndrome (hereditary nonpolyposiscancer), J. Med. Genet. 44 (2007) 353-362.

[14] J. Kollias, I.O. Ellis, C.W. Elston, R.W. Blamey, Prognostic significance of synchronous and metachronous bilateral breast cancer, World J. Surg. 25 (9) (2001) 1117-1124

[15] C.C. Gurin, M.G. Federici, L. Kang, J. Boyd, Causes and consequences of microsatellite instability in endometrial carcinoma, Cancer Res. 59 (January (2)) (1999) 462-466

[16] K. Suzuki, H. Matsubara, Recent advances in p53 research and cancer treatment, J. Biomed. Biotechnol. 13 (2011) 403-410.

[17] Vinnik IuA, V.N. Gorbenko, A.R. Vas'ko, E.V. Kikhtenko, V.V. Gargin, Incidence of anaplastic tumor in structure of other histologic forms of the thyroid gland cancer, Klin. Khir. (January (1)) (2014) 32-34.

[18] A. Romaniuk, M. Lyndin, Immune microenvironment as a factor of breast cancer progression, Diagn. Pathol. 10 (79) (2015).

[19] J.F. Lara, A.D. Thor, L.G. Dressler, G. Broadwater, I.J. Bleiweiss, S. Edgerton, D Cowan, L.J. Goldstein, S. Martino, J.N. Ingle, I.C. Henderson, L. Norton, E.P. Winer, C.A. Hudis, M.J. Ellis, D.A. Berry, D.F. Hayes, p53 Expression in node-positive breast cancer patients: results from the Cancer and Leukemia Group B 9344 trial (159905), Clin. Cancer Res. 17 (August (15)) (2011) 5170-5178.

[20] J.E. Chipuk, T. Moldoveanu, F. Llambi, M.J. Parsons, D.R. Green, The BCL-2 family reunion, Mol. Cell. 37 (February (3)) (2010) 299-310.

[21] X.M. Yin, Z.N. Oltvai, S.J. Korsmeyer, BH1 and BH2 domains of Bcl-2 are required for inhibition of apoptosis and heterodimerization with Bax, Nature 369 (1994) 321-333. 